\title{
The energy spectrum in a barotropic atmosphere
}

\author{
M. V. Kurgansky \\ Department of Geophysics, Faculty of Physical and Mathematical Sciences, University of Concepción, Chile \\ on leave from: A. M. Obukhov Institute of Atmospheric Physics, Russian Academy of Sciences, Moscow, Russia
}

Received: 26 September 2007 - Revised: 22 November 2007 - Accepted: 20 February 2008 - Published: 12 March 2008

\begin{abstract}
In a forced-dissipative barotropic model of the atmosphere on a spherical planet, by following mathematical techniques in (Thompson, P. D.: The equilibrium energy spectrum of randomly forced two-dimensional turbulence, Journal of the Atmospheric Sciences, 30, 1593-1598, 1973) but applying them in a novel context of the discrete spectrum on a rotating sphere, the "minus 2" energy spectrum for wavenumbers much greater than a characteristic wavenumber of the baroclinic forcing has been obtained if the forcing is taken in the simplest and most fundamental form. Some observation-based atmospheric kinetic energy spectra, with their slopes lying between "minus 2" and "minus 3" laws, are discussed from the perspective of the deduced "minus 2" energy spectrum.
\end{abstract}

\section{Introduction}

Globally, the atmosphere is permanently observed in the state of chaos, which serves as a background for individually better-distinguishable irregular processes of different temporal and spatial scales (the so called atmospheric circulation regimes). Chaotic dynamics of the atmospheric climate system occurring on its attractor (Lorenz, 1994) could be regarded as quasi-equilibrium in that sense that on the attractor we assume (and for certain atmospheric models it could strictly be proved (e.g., Dymnikov and Filatov 1997)) the existence of a unique stationary statistical distribution; that is an essential invariant measure on the attractor does exist. To characterize the attractor of the atmospheric climate system, we shall in this study focus on the steady equilibrium energy distribution between atmospheric disturbances of different spatial scales; this question has also fundamental implica-

Correspondence to: M. V. Kurgansky (kurgansk@udec.cl) tions for the problem of atmospheric predictability (Lorenz, 1969; see also Thompson, 1973).

Energy spectra are skillfully used for diagnostics of global atmospheric data and global atmospheric circulation model (GCM) outputs (see, e.g., Frederiksen et al., 2003, and references therein), usually with a clear reference to the famous "minus 3" spectral law. It was introduced by Kraichnan (1967), Leith (1968) and Batchelor (1969) (hereafter referred to as KLB) for the two-dimensional turbulence and proposed for the atmospheric quasi-geostrophic flows by Charney (1971) and later on by Gavrilin et al. (1972) (see Monin, 1990, and references therein). This spectrum is also distinguished within the predictability theory: for an energy spectrum which is less steep than "minus 3" the range of predictability is finite and intrinsically limited (Lorenz, 1969). Still, in atmospheric data the evidence for the "minus 3" law validity is not totally compelling. Most likely, this law is best applicable to upper tropospheric levels, whereas in the lower troposphere the observed atmospheric spectra are closer to the "minus 2" law (cf. Trenberth and Solomon, 1993; see also our Table 1).

By using the balance (in the ensemble averaged sense) of baroclinic forcing and viscous damping as constraints and under the presumably simplest assumption concerning the baroclinic forcing, we shall show in this note that the "minus 2" spectral law naturally emerges in a barotropic atmospheric model. We shall closely follow mathematical arguments by Thompson (1973), who intended to confirm the main predictions of KLB-theory for continuous spectra of the twodimensional turbulence, but shall do it in a novel context of barotropic non-divergent airflows on a rotating spherical Earth in the presence of both the baroclinic forcing and quite general viscous damping. The latter aggregates the Ekman friction, the Newtonian dissipation and the hyperviscosity of any order in one viscosity operator but necessarily includes a scale-selective viscosity term as its ingredient; that is, the case of only Ekman drag remains beyond the scope of this

Published by Copernicus Publications on behalf of the European Geosciences Union. 
Table 1. Slope of straight line fit to the kinetic energy spectrum from Trenberth and Solomon (1993) for waves $n=14$ to 40 for January 1988 (the global ECMWF analysis at T106 resolution) and from Boer and Shepherd (1983) for waves $n=14$ to 25 for January 1979 (First GARP Global Experiment, FGGE, wavenumbers only out to $n=32$ ). (Adapted from Trenberth and Solomon (1993); their Table 1).

\begin{tabular}{ccc}
\hline $\begin{array}{c}\text { Pressure } \\
(\mathrm{hPa})\end{array}$ & $\begin{array}{c}\text { Trenberth and Solomon } \\
(1993)\end{array}$ & $\begin{array}{c}\text { Boer and Shepherd } \\
(1983)\end{array}$ \\
\hline 100 & -2.2 & -2.2 \\
150 & -2.7 & -2.6 \\
200 & -3.0 & -3.0 \\
250 & -2.9 & -2.9 \\
300 & -2.9 & -2.6 \\
400 & -2.6 & -2.3 \\
500 & -2.6 & -2.1 \\
700 & -2.5 & -1.9 \\
850 & -2.1 & -1.7 \\
1000 & -1.9 & -1.5 \\
\hline
\end{tabular}

note. We shall argue that the difference between the deduced "minus 2" law and the classical "minus 3" spectrum by KLB can be explained for forced-dissipative flows by discrete nature of the spectrum on the spherical Earth.

\section{Forced flow in a dissipative barotropic atmosphere}

In spherical coordinates the governing absolute vorticity equation takes the form

$$
\begin{aligned}
& \frac{\partial}{\partial t} \nabla^{2} \psi+J\left(\psi, \nabla^{2} \psi+2 \Omega \cos \vartheta\right) \\
& =F-\mu \nabla^{2} \psi+\sum_{s} v_{s}(-1)^{s}\left[\nabla^{2 s}-(-2)^{s-1} \nabla^{2}\right] \psi,
\end{aligned}
$$

where $\psi$ is the streamfunction, $\nabla^{2}$ is the spherical Laplacian and

$J(A, B)=\frac{1}{a^{2} \sin \vartheta}\left(\frac{\partial A}{\partial \vartheta} \frac{\partial B}{\partial \lambda}-\frac{\partial B}{\partial \vartheta} \frac{\partial A}{\partial \lambda}\right)$

is the Jacobian, written for two arbitrary functions $A$ and $B$. Also, $\lambda$ is the longitude, $\vartheta$ the co-latitude, $\Omega$ the angular velocity of the Earth's rotation and $a$ the Earth radius. Below, the Earth radius is chosen to coincide with the unit of length, $a=1$. The time-independent function $F(\lambda, \vartheta)$ mimics the baroclinicity action. Also, a quite general frictional operator is considered in the right-hand-side of Eq. (1). The first term of this operator describes the Ekman friction with the coefficient $\mu$. In each summand of the subsequent sum, the order of the viscous operator has integer values, $s=2,3, \ldots$, and $v_{s}$ is the corresponding viscosity coefficient. The case in which $s=2$ corresponds to the common Newtonian viscosity with the coefficient $\nu_{2}$; the operator $\nabla^{2}\left(\nabla^{2}+2\right)$ strictly follows from the Navier-Stokes equations written down in spherical coordinates and the eigenfunction of $\nabla^{2}$ corresponding to the eigenvalue -2 and describing an arbitrary solid-body-like rotation of the atmosphere vanishes in $\nabla^{2}\left(\nabla^{2}+2\right)$. Therefore, the Newtonian viscosity does not affect the conservation of the angular momentum of the atmosphere. The 'hyperviscosity' operators of the order $s \geq 3$, used in Eq. (1), have the same property. However, an exact, unambiguous specification of the hyperviscosity operator of a high enough order, with regard to this property, is a more involved task and remains beyond the scope of this study. On the other hand, a wide variety of hyperviscosity operators, often without reference to the property in question, is currently used in numerical modeling studies. Therefore, as a "working compromise", the virtually simplest hyperviscosity operator of the given or$\operatorname{der} s$ is used in the work, which does not damp an arbitrary solid body rotation of the atmosphere. Note, that the additional terms in the above-discussed operators, $v_{s} 2^{s-1} \nabla^{2} \psi$, and the Ekman friction term in Eq. (1) can be combined in a single Rayleigh friction term $-\tilde{\mu} \nabla^{2} \psi$ with the coefficient $\tilde{\mu}=\mu-\sum_{s} 2^{s-1} v_{s}$, which in reality only slightly differs from $\mu$, because only very small $v_{s}$-values are commonly used in the atmosphere numerical modeling practice. In Eq. (1) and elsewhere, $\nabla^{3} \psi \equiv \nabla\left(\nabla^{2} \psi\right), \nabla^{4} \psi \equiv \nabla \cdot \nabla^{3} \psi$ and so on; $\nabla$ is the symbolic nabla operator on a sphere and a dot denotes the scalar product.

In an inviscid and unforced case, Eq. (1) allows for an infinite number of integrals of motion, called Casimir functions: any differentiable function of absolute vorticity integrated over the sphere is conserved, in particular, any integer power (moment) of absolute vorticity. All these Casimir functions except of the first and the second moment (the total vorticity and the enstrophy) are lost if spectral representation, and then truncation at a maximum wavenumber, are applied, as it is customarily used in numerical modeling.

In a statistically steady flow regime, the balance between energy generation due to the baroclinicity and energy dissipation due to the viscosity is assumed

$$
\begin{aligned}
\langle\mu & \left.\iint(\nabla \psi)^{2} d \sigma+\sum_{s} v_{s} \iint\left[\left(\nabla^{s} \psi\right)^{2}-2^{s-1}(\nabla \psi)^{2}\right] d \sigma\right\rangle \\
= & \left\langle-\iint \psi F d \sigma\right\rangle,
\end{aligned}
$$

where $d \sigma=\sin \vartheta d \vartheta d \lambda$ is the surface area element. Similarly, the enstrophy balance equation reads

$$
\begin{aligned}
\langle\mu & \left.\iint\left(\nabla^{2} \psi\right)^{2} d \sigma+\sum_{s} \nu_{s} \iint\left[\left(\nabla^{s+1} \psi\right)^{2}-2^{s-1}\left(\nabla^{2} \psi\right)^{2}\right] d \sigma\right\rangle \\
& =\left\langle\iint \nabla^{2} \psi F d \sigma\right\rangle .
\end{aligned}
$$

Angular brackets in Eqs. (2) and (3) denote the average over an ensemble of flow realizations on the global attractor of the system. Existence of this attractor is secured for the timeindependent forcing in Eq. (1) provided any scale-selective 
viscosity term with $s \geq 2$ is accounted for in Eq. (1); see Dymnikov and Filatov (1997) and references therein. Now, an invariant measure exists on the attractor, giving rise to a stationary statistical distribution on it. Under ergodicity assumptions, this stationary statistical distribution is unique and, also, the ensemble average in Eqs. (2) and (3) can be understood in the time-mean sense. A similar approach based on the balance between forcing and viscous damping as constraints to derive energy spectra was quite recently applied by Lynch and Verkley (2007).

Expanding $\psi$ in spherical harmonics $Y_{n}^{m}(\vartheta, \lambda)$ and using $G_{n}$ for denoting the expectation (expected) value of the baroclinic forcing contribution relevant to a subspace $\Sigma_{n}$ of dimension $2 n+1$, which is constituted by a set of functions $Y_{n}^{m}$ with the fixed lower index, or the degree, $n$, one has from Eqs. (2) and (3) that

$2 \mu \sum_{n=1}^{\infty} E_{n}+2 \sum_{s} v_{s} \sum_{n=1}^{\infty}\left[n^{s-1}(n+1)^{s-1}-2^{s-1}\right] E_{n}=\sum_{n=1}^{\infty} G_{n}$

and

$$
\begin{aligned}
2 \mu & \sum_{n=1}^{\infty} n(n+1) E_{n}+2 \sum_{s} v_{s} \sum_{n=1}^{\infty}\left[n^{s}(n+1)^{s}-2^{s-1} n(n+1)\right] E_{n} \\
& =\sum_{n=1}^{\infty} n(n+1) G_{n}
\end{aligned}
$$

respectively. Here, $E_{n}$ describes the expected value of kinetic energy which is relevant to $\Sigma_{n}$. If to consider the energy/enstrophy balance for different subspaces $\Sigma_{n}$ separately, then there must be energy/enstrophy fluxes between neighboring subspaces in order to maintain the steady statistical equilibrium. These internal fluxes cancel out each other after the summing in Eqs. (4) and (5) is made.

Our approach differs conceptually from the study by Thompson (1973) who investigated a stochastic system driven by a time-dependent random forcing. It is closer to the modern paradigm of internal climate variability, which is attributed to intrinsic non-linear chaotic dynamics of the climate system under time-constant external forcing conditions. However, the mathematical results which will be obtained based on Eqs. (6) and (7), see below, are remarkably insensitive to this conceptual difference, if compared to the analysis by Thompson (1973) based on his Eqs. (8) and (9).

In general cases, the baroclinic forcing $F(\lambda, \vartheta)$ may act over a range of wavenumbers $n$, which fall into the interval of baroclinic instability. Hereafter, we assume that $F$ is applied only at one specific wavenumber $n_{0}$ chosen from this range, i.e. it is the so-called monoscale forcing (see, Tran and Shepherd, 2004, and references therein) ${ }^{1}$. In the context of large-scale atmospheric dynamics, $n_{0}=6-8$ or even greater;

\footnotetext{
${ }^{1}$ For a time- and longitude-independent monoscale forcing $F(\vartheta)=F_{0} Y_{n_{0}}(\vartheta)$ Eq. (1) has an exact stationary solution. However, for sufficiently large forcing amplitude $F_{0}$ this steady zonal flow is unstable and a full spectrum of turbulent motions will develop due to non-linear interactions between modes.
}

in any case $n_{0} \gg 1$. Consistently with the monoscale forcing set-up, we retain only a single term $G_{n_{0}}$ corresponding to $n=n_{0}$ in the right-hand-sides of Eqs. (4) and (5), respectively, and assume that $G_{n_{0}}$ may parametrically depend upon $n_{0}$, generally speaking. We have now from Eqs. (4) and (5) that

$2 \mu \sum_{n=1}^{\infty} E_{n}+2 \sum_{s} v_{s} \sum_{n=1}^{\infty}\left[n^{s-1}(n+1)^{s-1}-2^{s-1}\right] E_{n}=G_{n_{0}}\left(n_{0}\right)$

and

$$
\begin{aligned}
& 2 \mu \sum_{n=1}^{\infty} n(n+1) E_{n}+2 \sum_{s} v_{s} \sum_{n=1}^{\infty}\left[n^{s}(n+1)^{s}-2^{s-1} n(n+1)\right] E_{n} \\
& \quad=n_{0}\left(n_{0}+1\right) G_{n_{0}}\left(n_{0}\right)
\end{aligned}
$$

Following Thompson (1973), we suppose that the energy spectrum has a universal form

$$
E_{n}=e_{0}\left(n_{0}\right) f\left(x_{n}\right), \quad x_{n}=\frac{n(n+1)}{n_{0}\left(n_{0}+1\right)},
$$

where function $f$ has to be specified. Equations (6) and (7) should satisfy for each and every $n_{0}$-value, viz. identically on $n_{0}$. Therefore, we take Eqs. (6) and (7) for two neighboring parameter values $n_{0}+1$ and $n_{0}$, say, substitute in them Eq. (8) taken for the same parameter values, and finally subtract the two resulting equations. Because $n_{0} \gg 1$ the relative increment in $n_{0}$ is small, $O\left(n_{0}^{-1}\right)$; therefore, we can with fair good accuracy replace the finite differences with differentials by formally imbedding integer $n_{0}$-values in the $n_{0}$-continuum; in this case, $x_{n}$ is also a continuous variable. Certainly, only integer $n_{0}$-values have physical meaning and will be retained in the final formulas.

Another way around would be using central finite differences, by subtracting the equations taken for $n_{0}+1$ and $n_{0}-1$, respectively, and by dividing the result by two. It may have certain methodological advantages by increasing an accuracy of our approximate approach; in particular, applying such a procedure to $n_{0}\left(n_{0}+1\right)$ yields $\left[\left(n_{0}+1\right)\left(n_{0}+2\right)-\left(n_{0}-1\right) n_{0}\right] / 2=2 n_{0}+1$, which equals to the formal derivative of $n_{0}\left(n_{0}+1\right)$ with respect to $n_{0}$, regardless of whether $n_{0} \gg 1$.

Keeping in mind what has been said above, we substitute Eq. (8) in Eq. (6), formally differentiate both parts of the resulting equation with respect to $n_{0}$ and use Eq. (6) once more to obtain

$$
\begin{aligned}
& \frac{d e_{0}}{d n_{0}} \sum_{n=1}^{\infty} K_{n} f\left(x_{n}\right)-\left(2 n_{0}+1\right) e_{0} \sum_{n=1}^{\infty} \frac{K_{n} f^{\prime}\left(x_{n}\right) x_{n}^{2}}{n(n+1)} \\
& \quad=e_{0} \frac{d \log G_{n_{0}}}{d n_{0}} \sum_{n=1}^{\infty} K_{n} f\left(x_{n}\right),
\end{aligned}
$$

where

$$
K_{n}=K_{n}\left(\mu, v_{2}, v_{3}, \ldots\right) \equiv 2 \mu+2 \sum_{s} v_{s}\left[n^{s-1}(n+1)^{s-1}-2^{s-1}\right] .
$$


The prime in Eq. (9) denotes differentiation with respect to $x_{n}$. Similarly, from Eqs. (7) and (8) we have

$$
\begin{aligned}
& \frac{d e_{0}}{d n_{0}} \sum_{n=1}^{\infty} n(n+1) K_{n} f\left(x_{n}\right)-\left(2 n_{0}+1\right) e_{0} \sum_{n=1}^{\infty} K_{n} f^{\prime}\left(x_{n}\right) x_{n}^{2} \\
& =e_{0}\left[n_{0}\left(n_{0}+1\right) \frac{d \log G_{n_{0}}}{d n_{0}}+2 n_{0}+1\right] \sum_{n=1}^{\infty} K_{n} f\left(x_{n}\right) x_{n} .
\end{aligned}
$$

We regard Eqs. (9) and (10) as a system of two linear algebraic equations for unknown variables $d e_{0} / d n_{0}$ and $e_{0}$. The condition for the existence of a nontrivial solution of this system is

$$
\begin{aligned}
& \frac{\sum_{n=1}^{\infty} n(n+1) K_{n} f\left(x_{n}\right)}{\sum_{n=1}^{\infty} K_{n} f\left(x_{n}\right)} \\
& =\frac{\left(2 n_{0}+1\right) \sum_{n=1}^{\infty} K_{n} f^{\prime}\left(x_{n}\right) x_{n}^{2}+\left[n_{0}\left(n_{0}+1\right) \frac{d \log G_{n_{0}}}{d n_{0}}+2 n_{0}+1\right] \sum_{n=1}^{\infty} K_{n} f\left(x_{n}\right) x_{n}}{\left(2 n_{0}+1\right) \sum_{n=1}^{\infty} \frac{K_{n} f^{\prime}\left(x_{n}\right) x_{n}^{2}}{n(n+1)}+\frac{d \log G_{n_{0}}}{d n_{0}} \sum_{n=1}^{\infty} K_{n} f\left(x_{n}\right)}
\end{aligned}
$$

Equations (6) and (7) imply, however, that the left-hand side of Eq. (11) equals to $n_{0}\left(n_{0}+1\right)$. Therefore, function $f$ is specified by the equation

$$
\sum_{n=1}^{\infty} K_{n}\left(\mu, \nu_{2}, v_{3}, \ldots\right) F\left(x_{n}\right)=0,
$$

where

$$
\begin{gathered}
F\left(x_{n}\right)=\left(2 n_{0}+1\right) x_{n}\left[f^{\prime}\left(x_{n}\right)\left(1-x_{n}\right)-f\left(x_{n}\right)\right] \\
+n_{0}\left(n_{0}+1\right) \frac{d \log G_{n_{0}}}{d n_{0}}\left(1-x_{n}\right) f\left(x_{n}\right) .
\end{gathered}
$$

Strictly speaking, the precise choice of the dependency $G_{n_{0}}\left(n_{0}\right)$ to be used in Eq. (13) should rely upon the observational/modeling results about the baroclinic instability in the real atmosphere, which however are not entirely conclusive or, at least, are not easy to be interpreted, in the given respect. Therefore, we shall first consider the conceptually simplest case when $G_{n_{0}}$ remains invariant, $G_{n_{0}}=$ const, when the wavenumber $n_{0}$ experiences variations within the range of atmospheric baroclinic instability. It yields $d G_{n_{0}} / d n_{0}=0^{2}$, hence the last right-hand-side term in Eq. (13) vanishes and from Eqs. (12) and (13) we obtain the condition for the existence of a nontrivial solution

$\sum_{n=1}^{\infty} K_{n}\left(\mu, \nu_{2}, \nu_{3}, \ldots\right)\left(2 n_{0}+1\right) x_{n}\left[f^{\prime}\left(x_{n}\right)\left(1-x_{n}\right)-f\left(x_{n}\right)\right]=0$.

\footnotetext{
${ }^{2}$ From the perspective of the theory of atmospheric general circulation, the assumption $G_{n_{0}}=G=$ const corresponds to the fixed rate of kinetic energy generation/dissipation per unit mass; in the real atmosphere $G \sim 5 \times 10^{-4} \mathrm{~m}^{2} \mathrm{~s}^{-3}$. It can alternatively be assumed that the atmospheric general circulation is functioning in the most efficient way and $G_{n_{0}}$ achieves its maximum stationary value $G$ for most rapidly growing baroclinic disturbances with wavenumbers $n_{0}=6-8$.
}

Since we are seeking for a universal energy spectrum, whose shape is independent on a specific form of the viscous operator in Eq. (1), Eq. (14) should be satisfied for every function $K_{n}\left(\mu, v_{2}, \nu_{3}, \ldots\right)$ and vanishing of this infinite sum is guaranteed only if its common member is zero, i.e. $f^{\prime}\left(x_{n}\right)\left(1-x_{n}\right)-f\left(x_{n}\right)=0$; hence,

$f\left(x_{n}\right)=\left\{\begin{array}{ll}C\left(x_{n}-1\right)^{-1}, & x_{n}>1 \\ C\left(1-x_{n}\right)^{-1}, & x_{n}<1\end{array}\right.$,

where $C>0$ is a constant. The distinction between the two cases, $x_{n}>1$ and $x_{n}<1$, respectively, is necessary because of the requirement $f\left(x_{n}\right)>0$. By introducing $x_{n}$ from Eq. (8) into Eq. (15) and considering only integer $n_{0}$-values, we obtain the spectral energy distribution

$E_{n}=\left\{\begin{array}{l}\frac{n_{0}\left(n_{0}+1\right) C e_{0}}{n(n+1)-n_{0}\left(n_{0}+1\right)}, \quad n>n_{0} \\ \frac{n_{0}\left(n_{0}+1\right) C e_{0}}{n_{0}\left(n_{0}+1\right)-n(n+1)}, \quad 1 \leq n<n_{0}\end{array}\right.$.

For the applied simplest forcing $G_{n_{0}}$ the model predicts the "minus 2" energy spectrum at $n \gg n_{0}$. In the opposite limit case of $1 \leq n \ll n_{0}$, Eq. (16) shows an approximate equipartition of the energy between subspaces $\Sigma_{n}$. As in Thompson (1973), the energy spectrum has singularity at $n=n_{0}$ and this peculiar property of the model is discussed in the paper just cited. The energy spectrum regularity could be retained if the energy were injected into the system, at minimum, at two neighboring wavenumbers.

As another tractable special case but of increasing complexity we consider

$G_{n_{0}}\left(n_{0}\right) \propto\left[n_{0}\left(n_{0}+1\right)\right]^{r}, \quad r>0$

whereas the previous case corresponds to $r=0$. We have from Eqs. (12) and (13) the condition for the existence of a nontrivial solution

$$
\begin{aligned}
& \sum_{n=1}^{\infty} K_{n}\left(\mu, \nu_{2}, v_{3}, \ldots\right)\left(2 n_{0}+1\right) \\
& \quad\left[f^{\prime}\left(x_{n}\right) x_{n}\left(1-x_{n}\right)-f\left(x_{n}\right) x_{n}+r f\left(x_{n}\right)\left(1-x_{n}\right)\right]=0, \\
& \text { or } f^{\prime}\left(x_{n}\right) x_{n}\left(1-x_{n}\right)-f\left(x_{n}\right) x_{n}+r f\left(x_{n}\right)\left(1-x_{n}\right)=0 \text { (see }
\end{aligned}
$$
above). Consequently,

$f\left(x_{n}\right)=\left\{\begin{array}{ll}C\left(x_{n}-1\right)^{-1} x_{n}^{-r}, & x_{n}>1 \\ C\left(1-x_{n}\right)^{-1} x_{n}^{-r}, & x_{n}<1\end{array}\right.$,

where $C>0$ is a constant. By introducing $x_{n}$ from Eq. (8) into Eq. (18) and considering only integer $n_{0}$-values, we obtain the spectral energy distribution

$E_{n}=\left\{\begin{array}{l}\frac{n_{0}\left(n_{0}+1\right) C e_{0}}{n(n+1)-n_{0}\left(n_{0}+1\right)} \cdot \frac{n_{0}^{r}\left(n_{0}+1\right)^{r}}{n^{r}(n+1)^{r}}, \quad n>n_{0} \\ \frac{n_{0}\left(n_{0}+1\right) C e_{0}}{n_{0}\left(n_{0}+1\right)-n(n+1)} \cdot \frac{r_{0}^{r}\left(n_{0}+1\right)^{r}}{n^{r}(n+1)^{r}}, \quad 1 \leq n<n_{0}\end{array}\right.$.

To specify $r$-value, we might for example, invoke a certain analogy with equilibrium statistical fluid dynamics (see also below) and make a special assumption that in the considered 
case of $n_{0} \gg 1$ the amplitude $G_{n_{0}}$ should be proportional to the dimension of subspace $\Sigma_{n_{0}}$,

$G_{n_{0}} \propto 2 n_{0}+1$.

It gives $r=1 / 2$ in Eq. (19) if to require, within the accuracy of the factor of two, the correspondence between Eqs. (17) and (20) for $n_{0} \gg 1$. However, direct substitution of Eq. (20) in Eq. (13) would lead to mathematical inconsistencies: variables $n_{0}$ and $x_{n}$ could not have been separated and a closed expression for the solution of Eq. (12) in terms of function $f$ and its derivative could not have been obtained. Now, formally likewise in Thompson (1973), who however investigated the continuous energy spectrum on an infinite plane, we obtain the "minus 3" spectrum for $n \gg n_{0}$; whereas, there is a hint on the "minus 1 " spectrum for $1 \leq n \ll n_{0}$. Interestingly, the latter spectrum appears in the study by Frederiksen and Sawford (1980) and Frederiksen et al. (1996) of equilibrium statistical mechanics for barotropic atmospheric flows over the rotating spherical Earth.

When Eq. (17) with $r=1 / 2$ is applied, then our Eqs. (6) and (7), on the one hand, and Thompson's (1973) basic Eqs. (8) and (9), on the other hand, are mathematically isomorphic, as far as it concerns the functional dependence of their right-hand-sides upon the scalar wavenumber, i.e. $\sqrt{n_{0}\left(n_{0}+1\right)}$ in our case. The very fact that we have obtained for $r=1 / 2$ the same energy spectrum as in Thompson (1973) proves a posteriori the consistency of applied approximate, asymptotic analysis.

Whereas the virtually most natural choice by Thompson (1973) of the baroclinic forcing on an infinite plane leads to the "minus 3" spectrum of KLB-theory, the simplest, "gauge-invariant", baroclinic forcing $G_{n_{0}}\left(n_{0}\right)=$ const on a sphere gives the $n^{-2}$-spectrum for large $n$. Therefore, the relationship between the continuous and the discrete spectra in forced-dissipative systems is not as straightforward, as it might seem. It includes a crucial dependence on the baroclinic forcing and could be highly sensitive to the way how the forcing is translated from the continuous into the discrete spectral variables.

In synthesis, in the frame of our model the question of energy spectral distribution is reduced to the problem of the baroclinic forcing $G_{n_{0}}\left(n_{0}\right)$ specification. Here, the "minus 2 " spectrum has a definite advantage over other possible energy spectra including the "minus 3" spectrum, since it is based upon the conceptually simplest assumption $G_{n_{0}}=$ const when virtually no baroclinic forcing parameters enter its definition. At the very least, the two spectra, "minus 3" and "minus 2", are seen as if they were placed on a similar, if not the same, conceptual footing.

\section{Discussion and concluding remarks}

Table 1, which is adapted from Trenberth and Solomon (1993) and also uses the results by Boer and Shepherd (1983), clearly shows that observed atmospheric spectra have slopes lying between the dependency $E_{n} \propto n^{-2}$, cf. Eq. (16), and the dependency, $E_{n} \propto n^{-3}$, which formally corresponds to KLB-theory for the two-dimensional turbulence. It is uneasy, in principle, to give a preference to any of these dependencies when looking at Table 1, though the later and more complete data by Trenberth and Solomon (1993) show systematic shift toward $E_{n} \propto n^{-3}$. Nevertheless, at the lowest atmospheric levels the obtained spectra in both cases are very close to the spectrum $E_{n} \propto n^{-2}$, see Eq. (16). Also, Lambert (1981) based on FGGE IIIa global data (January 1979, the same 10 isobaric levels as in Table 1) for the range of wavenumbers $14 \leq n \leq 25$ demonstrated strongly decreasing enstrophy spectral cascade and spectral slopes of -2.2 , -0.3 and -2.1 for the kinetic energy, the enstrophy and the available potential energy, correspondingly. These values are close to the scaling law $E_{n} \propto n^{-2}$. As a whole, the spectra $E_{n} \propto n^{-2}$ and $E_{n} \propto n^{-3}$ virtually serve as a lower and an upper bound, respectively, for the observed atmospheric spectra slopes (see, Table 1).

In order to get additional insight into the "minus 2" spectral law (this time, from the perspective differing to that of Sect. 2), let us consider a time-constant influx of enstrophy $G_{0}$, per unit atmospheric mass and with dimension of $\mathrm{s}^{-3}$, which is due to the baroclinic forcing applied within a narrow interval of wavenumbers $n$ centered at a wavenumber $n_{0}$. Both the width of the interval and the exact $n_{0}$-value are immaterial; they do not enter the final result. In a statistically steady regime, $G_{0}$ equals to the rate of enstrophy destruction due to viscosity. We recall that the total kinetic energy per unit mass, $E$, of a barotropic flow on a sphere is given by $E=\sum_{n=1}^{\infty} E_{n}$, where $E_{n}$ has dimension of $\mathrm{m}^{2} \mathrm{~s}^{-2}$ and describes the expected value of energy contribution coming from a subspace $\Sigma_{n}$. For a scale-selective dissipation in Eq. (1), viz. when accounting for at least one viscosity term with $s \geq 2$, we would have an inertial range of wavenumbers $n_{0} \ll n \leq n^{*}\left(v_{s}\right)$ with the nearly constant downscale enstrophy flux $G_{0}$. On dimensional grounds and in the spirit of KLBtheory we obtain

$E_{n}=C^{*} G_{0}^{2 / 3} a^{2}[n(n+1)]^{-1}$,

where $C^{*}$ is a non-dimensional constant. Here, the original Earth radius $a$ definition of Sect. 2 is used together with the fact that for a subspace $\Sigma_{n}$ the proper spatial scale squared is reciprocal to the magnitude of Laplace operator's eigenvalue, $n(n+1) / a^{2}$. We thus have formally obtained the "minus 2" energy spectrum as a discrete counterpart of the classical "minus 3" spectrum in KLB-theory dealing with 
continuous spectra ${ }^{3}$. Comparison between Eq. (16) taken at $n \gg n_{0}$ and Eq. (21) enables one to specify the functional dependence $e_{0}=e_{0}\left(n_{0}\right)$ which remained undetermined in Sect. 2. First, we have $C n_{0}\left(n_{0}+1\right) e_{0}=C^{*} G_{0}^{2 / 3} a^{2}$. Second, for a nearly monoscale forcing $G_{0} a^{2}=n_{0}\left(n_{0}+1\right) G_{n_{0}}$ and eliminating of $G_{0}$ between the above two formulas yields: $C e_{0}=C^{*}(a G)^{2 / 3}\left[n_{0}\left(n_{0}+1\right)\right]^{-1 / 3}$, where $G_{n_{0}}=G=$ const. The case of solely Ekman friction is more subtle and different sort of argument were needed to support the "minus 2" spectrum. They necessitate truncation of the spectral model at a maximum wavenumber $N \gg n_{0}$ and will be reported elsewhere. So, a pending theoretical task could be paraphrased as rather to explain why the observed spectra in the upper troposphere are algebraically steeper than the "minus 2 " law than to explain observed deviations from the "minus 3" spectrum (cf. numerical simulations of forced 2D turbulence which are referred to by Tran and Shepherd (2002) and definitely show energy continuous spectra algebraically steeper than the "minus 3" spectrum).

A remaining controversy concerning the fundamental significance of either the "minus 3" or the "minus 2" atmospheric energy spectrum on a spherical planet could partly be resolved if to accept that it is of importance whether to use the main results of KLB-theory obtained for continuous spectra and then to translate them into the terms of discrete spectra, or alternatively, to consider from the very beginning the discrete spectral distributions and, afterwards, to apply the physical arguments borrowed from KLB-theory.

Acknowledgements. I am grateful to R. Faulwetter and to an anonymous reviewer for many valuable suggestions and comments which led to significant improvements in the manuscript.

Edited by: U. Harlander

Reviewed by: R. Faulwetter and another anonymous referee

\section{References}

Batchelor, G. K.: Computation of the energy spectrum in homogeneous two-dimensional turbulence, Phys. Fluids, 12, Suppl. II, 233-239, 1969.

Baroud, C. N., Plapp, B. B., She, Z.-S., and Swinney, H. L.: Anomalous self-similarity in a turbulent rapidly rotating fluid, Phys. Rev. Lett., 88, 11, 114501, doi:10.1103/PhysRevLett.88.114501, 2002.
Boer, G. J. and Shepherd, T. G.: Large-scale two-dimensional turbulence in the atmosphere, J. Atmos. Sci., 40, 164-184, 1983.

Burgers, J. M.: Mathematical examples illustrating relations occurring in the theory of turbulent fluid motion, Hon. Ned. Acad. Wet. Vern. Eerste Sectie, D1, XVII(2), 1-53, 1939.

Charney, J. G.: Geostrophic turbulence, J. Atmos. Sci., 28, 1087 1095, 1971.

Dymnikov, V. P. and Filatov, A. N.: Mathematics of Climate Modeling, Birkhäuser, Boston, 1997.

Frederiksen, J. S. and Sawford, B. L.: Statistical dynamics of twodimensional inviscid flow on a sphere, J. Atmos. Sci., 37, 717$732,1980$.

Frederiksen, J. S., Dix, M. R., and Kepert, S. M.: Systematic energy errors and the tendency toward canonical distribution in atmospheric circulation models, J. Atmos. Sci., 53, 887-904, 1996.

Frederiksen, J. S., Dix, M. R., and Davies, A. G.: On effects of closure-based eddy diffusion on the climate and spectra of a GCM, Tellus, 55A, 31-44, 2003.

Gavrilin, B. L., Mirabel, A. P., and Monin, A. S.: On the energy spectrum of synoptic processes, Izv. Akad. Nauk. SSSR, Fiz. Atmos. Oceana, 8, 483-493, 1972.

Kraichnan, R. H.: Inertial ranges in two-dimensional turbulence, Phys. Fluids, 10, 1417-1423, 1967.

Lambert, S. J.: A diagnostic study of global energy and enstrophy fluxes and spectra, Tellus, 33, 411-414, 1981.

Leith, C. H.: Diffusion approximation for two-dimensional turbulence, Phys. Fluids, 11, 671-673, 1968.

Lorenz, E. N.: The predictability of a flow which contains many scales of motion, Tellus, 21, 289-307, 1969.

Lorenz, E. N.: The Essence of Chaos, University of Washington Press, Seattle, 1994.

Lynch, P. and Verkley, W.: Energy spectra from entropy principle, Proc. IUGG XXIV General Assembly - IAMAS, Abstract 5209, Perugia, Italy, 2-13 July 2007, available at: http: //www.iugg2007perugia.it/webbook/, 2007.

Monin, A. S.: Theoretical Geophysical Fluid Dynamics, Kluwer Academic Publishers, Dordrecht, The Netherlands, 1990.

Thompson, P. D.: The equilibrium energy spectrum of randomly forced two-dimensional turbulence, J. Atmos. Sci., 30, 1593 1598, 1973.

Tran, C. V. and Shepherd, T. G: Constraints on the spectral distribution of energy and enstrophy dissipation in forced twodimensional turbulence, Physica D, 165, 199-212, 2002.

Trenberth, K. E. and Solomon, A.: Implications of global atmospheric spatial spectra for processing and displaying data, J. Climate, 6, 531-545, 1993.

Zhou, Y.: A phenomenological treatment of rotating turbulence, Phys. Fluids, 7, 8, 2092-2094, 1995.

\footnotetext{
${ }^{3}$ The "minus 2" law is reminiscent of an early "equilibrium statistical fluid mechanical" prediction by Burgers (1939) for his simple one-dimensional fluid system. The " -2 " scaling law emerges in turbulent rapidly rotating fluids (Zhou, 1995; see direct experimental support in Baroud et al. (2002)).
} 